\title{
Double-patch repair of left ventricular rupture after mitral valve replacement
}

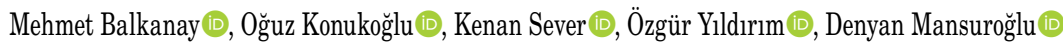 \\ Department of Cardiovascular Surgery, Yeni Yüzyll University, Private Gaziosmanpaşa Hospital, Istanbul, Turkey \\ Received: July 09, 2021 Accepted: September 02, 2021 Published online: November 12, 2021
}

\begin{abstract}
Left ventricular rupture is a rare, but catastrophic complication of mitral valve surgery. Herein, we describe the double-patch repair technique that can be applied in case of left ventricular rupture after mitral valve replacement. Using this technique, the first pericardial patch is positioned inside the endocardium, and the second pericardial patch is positioned outside the epicardium creating a double-layer closure of the defect.
\end{abstract}

Keywords: Double-patch repair, left ventricle, mitral valve surgery, rupture.

Left ventricular rupture after mitral valve replacement was first reported by Roberts and Morrow $^{[1]}$ in 1967 in a postmortem study. Since then, many surgeons have sought a solution to this mortal surgical approach. ${ }^{[2-7]}$ Left ventricular ruptures after mitral valve replacement are rare, but fatal complications. The incidence varies between 0.24 and $2 \%$ in the literature. ${ }^{[5-8]}$ Surgical mortality is very high up to 50 to $100 \%)^{[5,7,9]}$ Late ruptures result in pseudoaneurysms and, therefore, treatment of late ruptures is more favorable. Herein, we describe the double-patch repair technique to manage this difficult situation.

\section{SURGICAL TECHNIQUE}

A 53-year-old male patient underwent mitral valve replacement with a $29-\mathrm{mm}$ bioprosthesis for mitral valve stenosis and single coronary artery bypass for right coronary artery stenosis. At the end of the operation, while closing the sternum, massive bleeding started from the posterior of the heart. The patient was immediately re-cannulated and cardiopulmonary bypass $(\mathrm{CPB})$ was re-initiated. Bleeding was detected from the posterior wall of the left ventricle. The digital examination revealed that the strut of the bioprosthesis ruptured the posterior wall of the left ventricle. After cross-clamping the aorta, diastolic cardiac arrest was induced using antegrade blood cardioplegia. The mitral bioprosthesis was left in situ, and an external repair technique was chosen.
The posterolateral portion of the heart was exposed by two hanging sponges. We prepared two autologous elliptical pericardial patches of $2 \times 3 \mathrm{~cm}$ in dimensions. Pericardial patches were treated with $0.9 \%$ glutaraldehyde solution for $2 \mathrm{~min}$. To adjust the size of the pericardial patches, they should be larger than the defect of the ruptured area. In this manner, the patches are healthy sutured avoiding tears of the fragile ruptured ventricular area.

First, 3/0, $25 \mathrm{~mm}$ double-needle non-absorbable Ticron ${ }^{\circledR}$ polyester U sutures (Sherwood-Davis \& Geek, MI, USA) were passed through the first pericardium (Figure 1). The same sutures were, then, passed through the endocardial, myocardial, and epicardial layers by appropriate intervals, in an inside-out manner, $1.5 \mathrm{~cm}$ distant to the ruptured area (Figure 2). The first pericardial patch was positioned to the endocardial face of the ventricle leaving the needles outside the pericardium (Figure 3). The needles outside the epicardium were passed from a second pericardial patch with the same dimensions

Corresponding author: Oğuz Konukoğlu, MD. Yeni Yüzyıl Üniversitesi, Özel Gaziosmanpaşa Hastanesi, Kalp ve Damar Cerrahisi Kliniği, 34245 Gaziosmanpaşa, İstanbul, Türkiye.

Tel: +90 505 - 8689732 e-mail: oguzkonukoglu@yahoo.com

Citation:

Balkanay M, Konukoğlu 0, Sever K, Yıldırım Ö, Mansuroğlu D. Double-patch repair of left ventricular rupture after mitral valve replacement. Cardiovasc Surg Int 2021;8(3):166-170. 
and this second patch was positioned on the epicardial surface of the ventricle creating a double-layer repair of the defect (Figure 4). In between the two patches, an absorbable collagen hemostatic sponge $\left(\right.$ Helistat $^{\circledR}$, Integra Life Sciences Inc., NJ, USA) was placed and $1 \mathrm{~mL}$ tissue adhesive (Preveleak ${ }^{\circledR}$, Baxter Healthcare Corp., CA, USA) was injected for reinforcement and prevention of leakage. Immediately after the adhesive dried, all the sutures were tied (Figures 5 and 6).

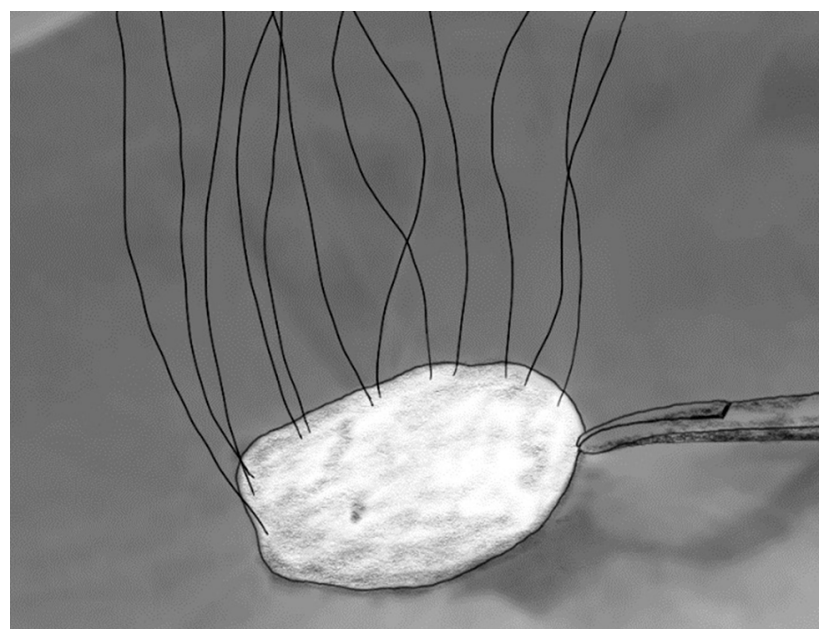

Figure 1. Double-needle U-sutures were passed through the first pericardial patch.

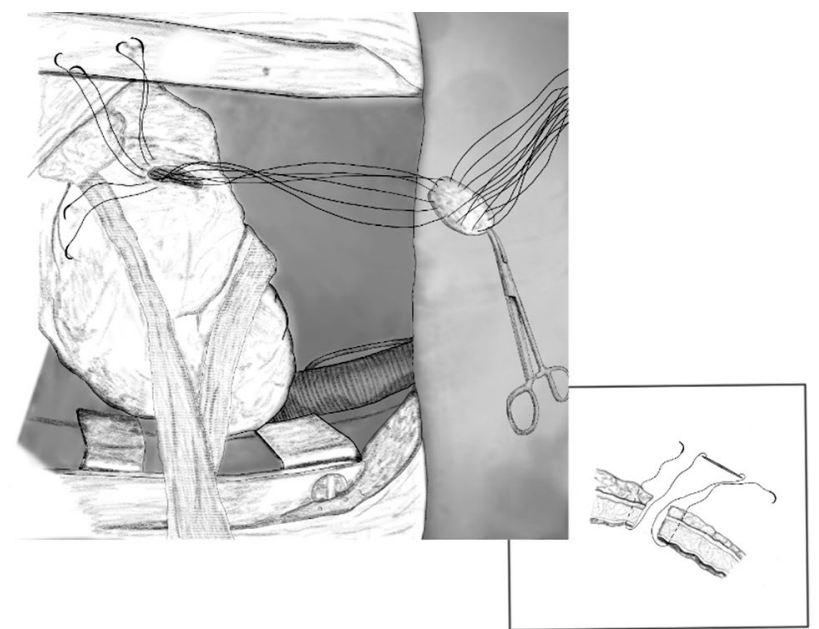

Figure 2. Double-needle U-sutures were, then, passed through the myocardium in an inside-out manner.

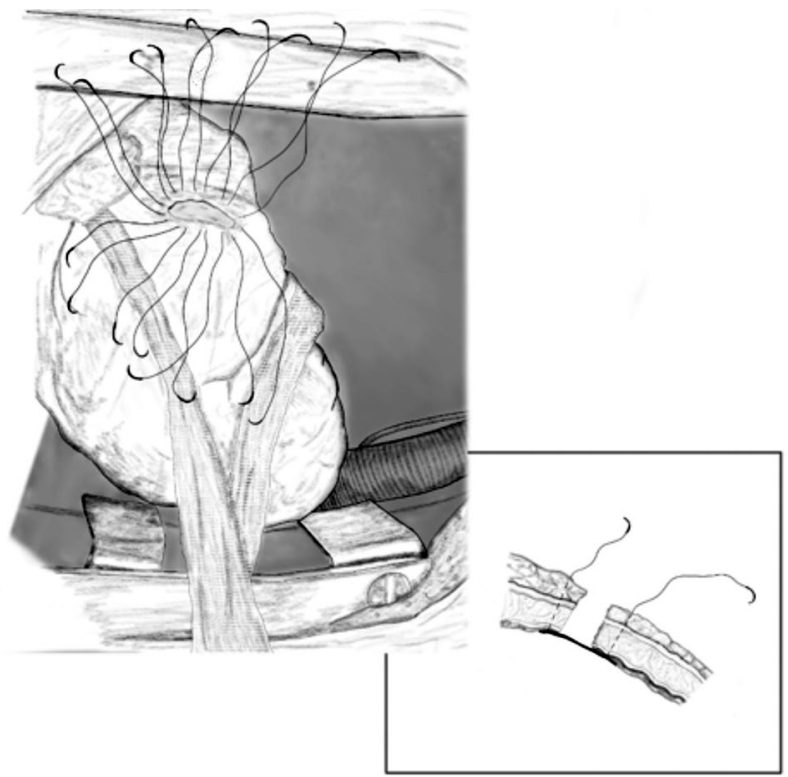

Figure 3. The first pericardial patch was positioned to the endocardial face of the ventricle, leaving the needles outside the epicardium.

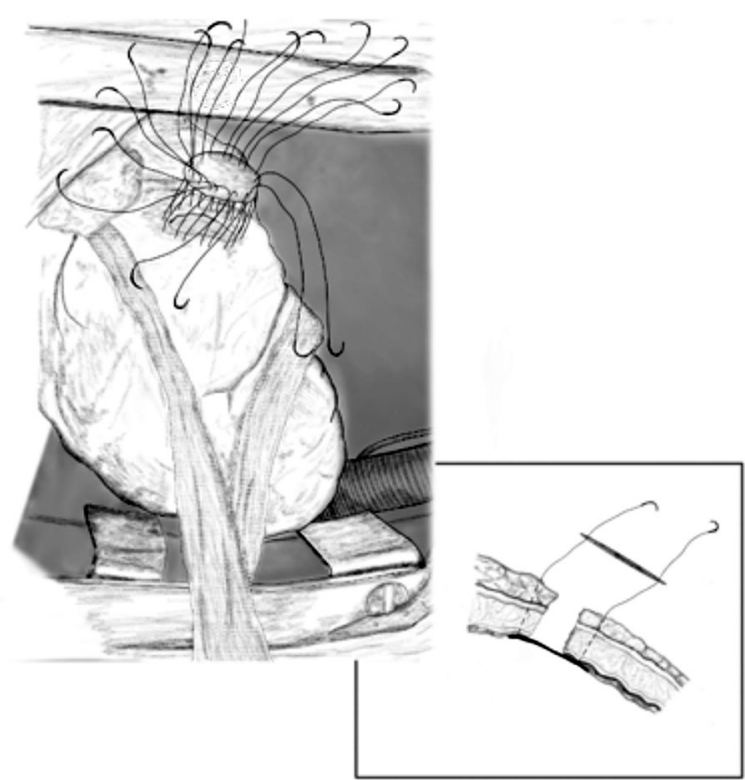

Figure 4. The needles outside the epicardium were passed from a second pericardial patch positioned on the epicardial surface of the ventricle creating a double-layer repair of the defect. 


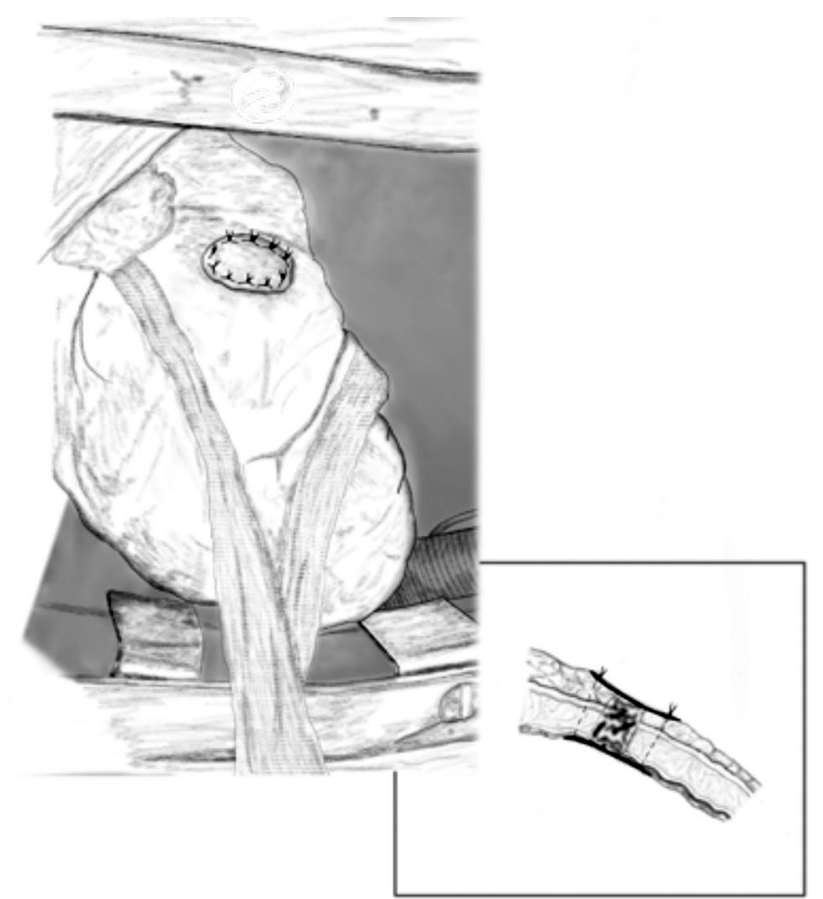

Figure 5. All the sutures were tied and the defect was securely closed.

The patient was weaned from CPB with intraaortic balloon and inotropic support and discharged on Day 11, after intensive care unit (ICU) stay for four days.

\section{Types of ventricular rupture after mitral valve surgery}

Classically, there are three types of ventricular rupture after mitral valve surgery. Type 1 lesion originates between posterior atrioventricular sulcus along the posterior mitral annulus. These are the most common lesions..$^{[2,6,7]}$ Type 2 lesion originates from the roots of papillary muscles due to aggressive excision of the anterolateral and posteromedial papillary muscles, ${ }^{[10]}$ in case of mitral valve replacement, chordal transfer, or excess and deep sutures in tissue. The primary reason for this complication is the chords' deep excision and the discordance between the artificial valve and the annulus. ${ }^{[1]}$ Eventually, ventricular region cannot withstand systolic contractions, and the small tear in the epicardium grows gradually. Type 3 lesion occurs between the type 1 lesion, atrioventricular groove, and type 2 lesion. ${ }^{[7]}$ This rupture can be caused by traumatic reasons, as well as by the misuse of scissors and scalpel, or traumatic effect of aspirator

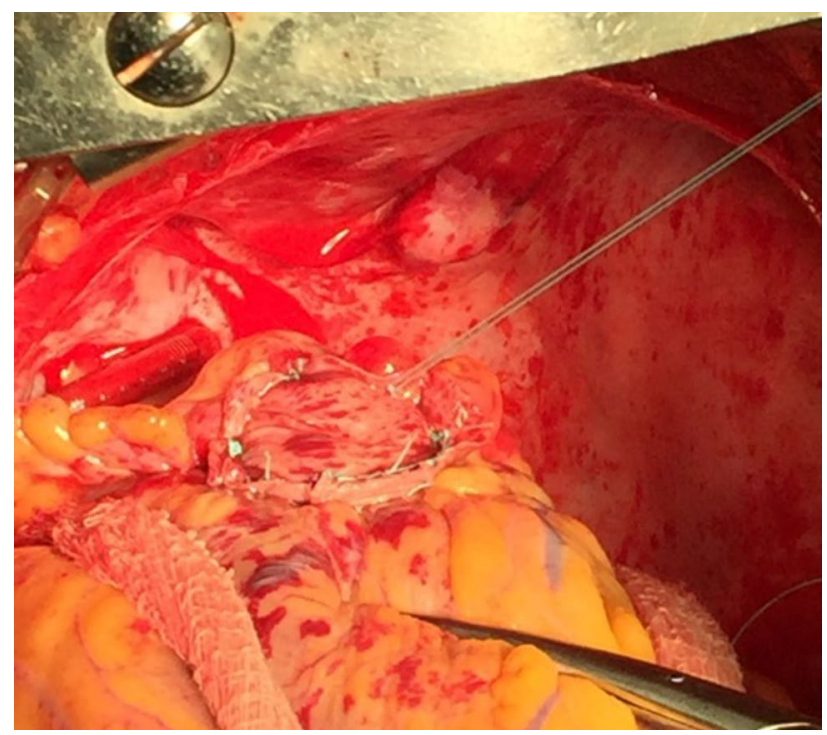

Figure 6. Final image of the double-patch repair.

tips. Beside these three classical sites, the rupture of the left ventricular outflow tract, most probably due to the struts of the mitral bioprosthesis, was also described in some cases. ${ }^{[6]}$

Risk factors for the formation of left ventricular wall ruptures are mainly ischemic heart disease, valve replacement based on annular abscess in case of infectious endocarditis, advanced age, chronic renal failure, left ventricular diastolic diameter less than $50 \mathrm{~mm},{ }^{[6,7,12]}$ posterior annular calcification, ${ }^{[12]}$ previous interventional cardiology applications for the mitral valve, redo heart surgery surgical dissection of the posterolateral wall, emergency intervention, calcified annulus, ${ }^{[13]}$ rigidity of the vent cannulas to be used to decompress the left heart and its' placement from the left ventricular apex ${ }^{[6]}$ female sex, low body mass index, mitral stenosis, small left ventricular cavity, ${ }^{[14]}$ excessive resection in operations, the use of a larger frame heart valve, high-profile valves, high-profile sharp-tipped struts, the technique of using deep sutures, creating excessive annular tension, unintentional injuries, and extreme stresses due to insufficient diastolic arrest. ${ }^{[6,7]}$

\section{DISCUSSION}

Left ventricular wall ruptures occur in the posterior and posterolateral wall and, classically, there are three different types according to the location of the rupture. Reports of posterior wall ruptures caused 
by strut positioning and their high incidence in the small left ventricular cavities ${ }^{[14]}$ lead the companies to reduce the strut profiles and lead the surgeons to pay attention, while positioning bioprosthesis struts with good results. ${ }^{[4]}$ The concept of "untethered loop" was introduced by Cobbs et al. ${ }^{[15]}$ The suture technique applied to the posterior annulus is crucial. Sutures from both the annulus and prosthetic valve should be positioned equally spaced, and the sutures should be reciprocal. Asymmetrical and uneven spaced suture positioning may cause either leakage or rupture in the later period. ${ }^{[4]}$

In the injured area, first dissection and hematoma develop in the myocardium and, then, rupture. ${ }^{[9,12]}$ In redo cases, the main reason is the deep cut of tissue through the annulus during the removal of the valve. ${ }^{[12]}$ However, in redo cases, this area is very adherent, and the attached posterior wall may be partially protective. Although acute left ventricular ruptures are not seen in these cases, pseudoaneurysms may develop in the late period. The repair of late pseudoaneurysms is easier, and the results are more satisfactory.

In the publication of Cabrol in 1984, Lillehei reported that none of the 2,100 cases had ventricular wall rupture for 20 years due to the protection of the posterior leaflet. ${ }^{[16]}$ In the series of Deniz et al., ${ }^{[13]}$ no ventricular wall rupture was observed in patients with preserved posterior leaflet. Karlson et al. ${ }^{[14]}$ also agreed with this opinion. In our practice, we also preserve the posterior leaflet in primary cases and bioprostheses. If cardiopulmonary resuscitation is needed in a patient with mitral valve prosthesis, we recommend a soft cardiac massage to avoid the rupture of left ventricle. It is not possible to preserve the posterior leaflet in all cases. However, it should be preserved in all eligible patients. Deep excision of the posterior annulus and deep hard decalcification should be avoided. The appropriate size of valves should be selected, sutures from both annulus and prosthetic valve should be equally spaced and reciprocal.

Treatment of left ventricular rupture is challenging in many ways. The left ventricular muscle tissue easily splits and does not support the sutures. ${ }^{[6]}$ Regardless of the type of rupture, it is difficult to access and vision is limited. ${ }^{[6]}$ Surgical suturing and positioning of the heart is also very difficult, when repair under a beating heart is chosen. Repair techniques on the beating heart can lead to elongation of the tear widening the ruptured area. Repairing the ventricle by decompressing the heart under $\mathrm{CPB}$ and cross-clamping the aorta is the most reliable way. ${ }^{[5]}$

The reasons that make the situation more difficult are sutures extending to the ventricle's endocardium that may damage the mitral bioprosthesis and the circumflex artery and its branches, inducing acute myocardial infarction (MI). ${ }^{[17]}$ Sutures may occlude the coronary sinus or cause stenosis. Malignant arrhythmias may develop in the following period. The surgical technique aims to cover the ruptured area with patches and pass the sutures through the intact myocardial tissue by a wide external patch covering the laceration and hematoma area. ${ }^{[10]}$ Also, the major branches of the circumflex artery must be protected. ${ }^{[6]}$ In type 1 tears, the great vein of the coronary sinus should not be damaged. In the double-patch repair or sandwich technique, sutures must pass through non-damaged tissue. If there is a doubt about the coronary arteries injury, revascularization of the suspected vessel is needed. Successful cases have been described in the literature using the posterior leaflet as support in type 1 ruptures. ${ }^{[6]}$

Repair with a buttressed suture technique using a felt strip is recommended in type 1 ventricular rupture. Both internal and extracardiac patching techniques are suggested for type 2, 3, and mixed ruptures. ${ }^{[6]}$ Another method is to support the surgical repair of the torn region with adhesives such as bio-glue. ${ }^{[7,9]}$ To create a bloodless environment in the 2 -min period in which adhesives are used, the total circulatory arrest may be needed. Adhesives alone do not form the basis of repair, but support the surgical technique. ${ }^{[9]}$ The chance of encountering left ventricular wall rupture varies between clinics and even between surgeons in a single clinic.

If the rupture occurs in the operating room, it is easier to re-start CPB, and the problem is easier to manage. However, if it occurs during the postoperative period, the diagnosis is complex and there may not be enough time for stabilization.

Two main routes of repair the left ventricular rupture have been defined as internal and external. ${ }^{[5,14]}$ The method we describe has an external approach, and it differs from the sandwich method 2 in terms of suture technique and placement of the pericardial patches.

In the internal repair, the lesion is exposed through atriotomy. However, if mitral valve repair 
is applied, it is impossible to perform the internal repair without removing the valve. The results of the external technique with $\mathrm{CPB}$ are better than the internal technique. ${ }^{[5,14]}$ The results of repair without $\mathrm{CPB}$ are even worse. ${ }^{[5,12]} \mathrm{We}$ do not recommend autotransplantation, which is the last step of the algorithm created by Sersar and Jamjoom ${ }^{[6]}$

In the present case, the patient underwent bioprosthetic valve implantation, and injury of the left ventricle due to the bioprosthesis struts occurred. The ventricle was repaired with the double-patch repair technique. Eventually, the patient was discharged on postoperative Day 11 after ICU stay for four days. We also used this technique in two more patients previously. Both of them were left ventricular free wall rupture due to ischemic MI. After the operation, bleeding stopped and both patients were discharged. All three patients weaned from the CPB with inotropic support. Two patients needed intra-aortic balloon support.

We also suggest the double-patch repair with a similar technique in patients with post-MI ventricular septal defect. ${ }^{[18]}$

In conclusion, preventive measures should be taken to avoid the occurrence of left ventricular rupture after mitral valve replacement, particularly in patients with predisposing factors. In case of left ventricular rupture, repair of the defect on $\mathrm{CPB}$ with cross-clamping the aorta protects the left ventricular functions, thereby avoiding excessive blood loss. The double-patch repair technique secures the suture lines and strengthens the jeopardized area with better results in this difficult situation to handle complication.

\section{Declaration of conflicting interests}

The authors declared no conflicts of interest with respect to the authorship and/or publication of this article.

\section{Funding}

The authors received no financial support for the research and/or authorship of this article.

\section{REFERENCES}

1. Roberts WC, Morrow AG. Causes of early postoperative death following cardiac valve replacement. Clinicopathologic correlations in 64 patients studied at necropsy. J Thorac Cardiovasc Surg 1967;54:422-37.

2. Sezer H, Isık O, Balkanay M, Berki T YC. 1-Left ventricular posterior wall rupture after mitral valve replacement. Kosuyolu Hear J 1990;1:54-7.
3. Lee ME, Tamboli M, Lee AW. Use of a sandwich technique to repair a left ventricular rupture after mitral valve replacement. Tex Heart Inst J 2014;41:195-7.

4. Spencer FC, Galloway AC, Colvin SB. A clinical evaluation of the hypothesis that rupture of the left ventricle following mitral valve replacement can be prevented by preservation of the chordae of the mural leaflet. Ann Surg 1985;202:673-80.

5. Costa MA, Laforga FC, Maftum JA, Favaro MG. Use of bovine pericardium and sutureless biological glue in left ventricular rupture after mitral valve replacement, five years of follow-up. Braz J Cardiovasc Surg 2015;30:673-5.

6. Sersar SI, Jamjoom AA. Left ventricular rupture post mitral valve replacement. Clin Med Cardiol 2009;3:10113.

7. Masroor S, Schor J, Carrillo R, Williams DB. Endoventricular pocket repair of type I myocardial rupture after mitral valve replacement: A new technique using pericardial patch, Teflon felt, and BioGlue. Ann Thorac Surg 2004;77:1439-41.

8. Wolpowitz A, Barnard MS, Sánchez HE, Barnard CN. Intraoperative posterior left ventricular wall rupture associated with mitral valve replacement. Ann Thorac Surg 1978;25:551-4.

9. Mejia R, Thomson DS. A new technique for repair of atrioventricular disruption complicating mitral valve replacement. Ann Thorac Surg 2003;75:1973-4.

10. Azariades M, Lennox SC. Rupture of the posterior wall of the left ventricle after mitral valve replacement: Etiological and technical considerations. Ann Thorac Surg 1988;46:491-4.

11. Zhang HJ, Ma WG, Xu JP, Hu SS, Zhu XD. Left ventricular rupture after mitral valve replacement: A report of 13 cases. Asian Cardiovasc Thorac Ann 2006;14:26-9.

12. Treasure RL, Rainer WG, Strevey TE, Sadler TR. Intraoperative left ventricular rupture associated with mitral valve replacement. Chest 1974;66:511-4.

13. Deniz H, Sokullu O, Sanioglu S, Sargin M, Ozay B, Ayoglu $\mathrm{U}$, et al. Risk factors for posterior ventricular rupture after mitral valve replacement: Results of 2560 patients. Eur J Cardiothorac Surg 2008;34:780-4.

14. Karlson KJ, Ashraf MM, Berger RL. Rupture of left ventricle following mitral valve replacement. Ann Thorac Surg 1988;46:590-7.

15. Cobbs BW, Jr, Hatcher CR, Jr, Craver JM, Jones EL, Sewell CW. Transverse midventricular disruption after mitral valve replacement. Am Heart J 1980;99:33-50.

16. David TE, Burns RJ, Bacchus CM, Druck MN. Mitral valve replacement for mitral regurgitation with and without preservation of chordae tendineae. J Thorac Cardiovasc Surg 1984;88:718-25.

17. Izzat MB, Smith GH. Rupture of left ventricle after mitral valve repair: Case report and new technique of repair. $\mathrm{Br}$ Heart J 1993;69:366-7.

18. Balkanay M, Eren E, Keles C, Toker ME, Guler M. Doublepatch repair of postinfarction ventricular septal defect. Tex Heart Inst J 2005;32:43-6. 